\title{
VALOR PRONÓSTICO DE LA PLOIDÍA DEL ADN Y LA MORFOMETRÍA NUCLEAR EN EL CÁNCER DE PRÓSTATA METASTÁSICO
}

\author{
J.M. MARTÍNEZ JABALOYAS*, A. JIMÉNEZ SÁNCHEZ**, J.L. RUIZ CERDÁ*, \\ S. SANZ CHINESTA*, A. SEMPERE***, J.F. JIMÉNEZ CRUZ* \\ *Servicio de Urología. **Servicio de Anatomía Patológica. ***Servicio de Hematología. \\ Hospital Universitario La Fe. Valencia. \\ Actas Urol Esp. 28 (4): 298-307, 2004
}

\section{RESUMEN}

VALOR PRONÓSTICO DE LA PLOIDÍA DEL ADN Y LA MORFOMETRÍA NUCLEAR EN EL CÁNCER DE PRÓSTATA METASTÁSICO FUNDAMENTO: Conocer el valor pronóstico de la ploidía del ADN y la morfometría nuclear determinadas en el foco tumoral primario en pacientes afectos de cáncer de próstata con metástasis óseas.

MÉTODOS: Estudio retrospectivo sobre 54 pacientes con adenocarcinoma de próstata y metástasis óseas tratados con supresión androgénica. En todos ellos se realizó análisis de ADN (citometría de flujo) sobre tejido tumoral desparafinado y estudio de morfometría nuclear. También se analizó la edad, la puntuación de Gleason, la categoría T, el hematocrito, el nivel de albúmina sanguínea, las fosfatasas alcalinas, fosfatasas ácidas prostáticas, y el número de focos metastásicos que aparecían en el rastreo. Se realizaron estudios univariantes y multivariantes de supervivencia mediante el método de Kaplan-Meier y de riesgos proporcionales de Cox, respectivamente. Finalmente, realizamos un análisis de regresión logística multivariante para los factores pronósticos clásicos y otro en el que se añadieron los experimentales (ploidia de ADN y morfometria nuclear), con la intención de conocer qué factores pueden predecir el que un paciente alcance la mediana de supervivencia y en qué medida los factores experimentales mejoran dicha predicción.

RESULTADOS: En el análisis univariante de supervivencia tuvieron carácter pronóstico la categoría T, el nivel de albúmina, las fosfatasas alcalinas, la puntuación de Gleason, el rastreo óseo, la ploidía del ADN y el área nuclear media. En el multivariante únicamente aportaron información pronóstica independiente la puntuación de Gleason, el rastreo óseo, el área nuclear media y la ploidía del ADN. En el estudio de regresión logística para factores pronósticos clásicos únicamente el Gleason tiene capacidad predictiva (sensibilidad $89,3 \%$, especificidad 64\%), mientras que al introducir los factores experimentales también el rastreo óseo y la ploidía del ADN son significativos (sensibilidad $90 \%$ y especificidad $72 \%$ ).

CONCLUSIONES: El estudio de contenido de ADN y de morfometría nuclear del foco tumoral primitivo aporta información pronóstica independiente en el análisis de supervivencia para el cáncer de próstata metastásico. Sin embargo, la escasa mejora en la capacidad predictiva sobre los factores pronósticos clásicos cuestionan su utilidad en la práctica clínica.

PALABRAS CLAVE: Cáncer de próstata. Metástasis. Ploidía del AND. Núcleo celular. Factores pronósticos. Análisis multivariante.

\section{ABSTRACT}

PROGNOSTIC VALUE OF DNA PLOIDY AND NUCLEAR MORPHOMETRY IN METASTATIC PROSTATE CANCER

PURPOSE: To assess the prognostic value of DNA ploidy and nuclear morphometry in metastatic prostate cancer after androgenic deprivation treatment.

METHODS: Fifty four patients with prostate cancer and bone metastases who had undergone androgenic suppression treatment were retrospectively studied. The deoxyribonucleic acid (DNA) content was analysed by flow cytometry. Nuclear morphometry characterized 14 nuclear descriptors. The study also included age, Gleason score, T classification, haematocrite, serum albumin, serum alkaline phosphatase, serum prostatic acid phosphatase and the amount of metastatic foci detected during radioisotope bone scan. Univariate survival analyses were performed and Cox's proportional hazards model was used to identify significant prognostic factors. To assess how the experimental factors improve the capacity of the classical factors for predicting the patients who reach median survival, logistic regression multivariate analysis was performed for the classical prognostic factors only and after added experimental variables (DNA content and Nuclear Area).

RESULTS: The univariate survival analyses assigned a prognostic value to $\mathrm{T}$ category, level of albumin, alkaline phosphatase, Gleason score, bone scan, DNA ploidy and mean nuclear area. In the case of the Cox regression model only Gleason score, bone scan, mean nuclear area and DNA ploidy provided independent prognostic information. In logistic regression for classic prognostic factors only Gleason score is significant (sensibility $89,3 \%$, specificity $64 \%$ ). However, when the experimental factors are added, in addition to Gleason score, radioisotope bone scan and DNA ploidy are of prognostic value (sensibility $90 \%$ and specificity $72 \%$ ).

CONCLUSIONS: The study of DNA content and nuclear morphometry in the primitive tumor provides independent prognostic information in survival analysis for patients with metastatic prostate cancer. However, there is limited improvement with respect to the classical factors in predicting survival. This questions its utility in the daily clinical usage. 
$\mathrm{E}$ s importante conocer la posible evolución de un paciente portador de una neoplasia, con el fin de planificar adecuadamente la estrategia terapéutica. Partiendo de la base de que los factores pronósticos clásicos, como el grado histológico y el estadio, no permiten realizar predicciones individuales sobre el comportamiento del carcinoma prostático, es necesario buscar nuevas características que mejoren la capacidad de predecir su evolución. Una vez que se han desarrollado metástasis, las probabilidades de curación son prácticamente nulas con una gran variabilidad pronóstica. Por otra parte las características de las metástasis pueden ser diferentes a las del tumor primario que las originaron y generalmente, el material analizado procede de este último, por lo que es arriesgado predecir que las características del tumor primario influyan en la evolución general de la masa tumoral, ya que aunque se ha mostrado que con la progresión tumoral aumenta la indiferenciación histológica ${ }^{1}$, existen controversias sobre la correlación en la ploidía entre el tumor primario y las metástasis, de tal forma que mientras hay autores que reportan una alta correlación ${ }^{2}$, otros describen una elevada discordancia $^{3}$. Además, sería muy difícil analizar las características de cada uno de los focos metastásicos, por lo que nos interesa encontrar factores clínicos y tumorales, fáciles de determinar, que nos definan la evolución que tendrá un determinado paciente.

La finalidad de nuestro estudio es precisar el valor pronóstico de la ploidía del $\mathrm{ADN}$ y de la morfometría nuclear, determinadas en el tumor primario, en un grupo de pacientes afectos de cáncer de próstata metastásico. Por otra parte, intentamos analizar la capacidad predictiva de los factores clínicos e histológicos clásicos (estadiaje tumoral, determinaciones analíticas y puntuación de Gleason) y conocer en qué medida los factores experimentales (ploidía de ADN y morfometría nuclear) mejoran dicha capacidad predictiva.

\section{MATERIAL Y MÉTODO}

Estudio retrospectivo en el que se incluyeron 54 pacientes diagnosticados de adenocarcinoma de próstata metastásico y tratados con bloqueo androgénico en el periodo comprendido entre 1975 y 1988.
La información del estadio se obtuvo a partir de las exploraciones clínicas y complementarias practicadas: analíticas sanguíneas, ecografía transrectal y abdominal, Rx tórax, TAC abdominopélvico y rastreo óseo. No hay cuantificación del PSA porque aún no se había introducido clínicamente en el momento del diagnóstico. Los resultados de los análisis de laboratorio fueron estratificados en 2 para el estudio estadístico: normales y alterados. Se ha utilizado la clasificación TNM de $1997^{4}$, estadiando a los pacientes según la categoría $T$. No distinguimos entre pacientes Mla y M1b y excluimos a los pacientes con metástasis viscerales. No se valoró la categoría $\mathrm{N}$ para la clasificación clínica, de forma que no se distinguió entre pacientes NO y N1. Según el resultado del rastreo óseo dividimos a los pacientes en 3 grupos: en el primero se incluían aquellos que presentaban menos de 6 focos metastásicos en el rastreo, en el segundo los que presentaban más de 6 focos y un tercero con los que tenían afectado más del $75 \%$ del esqueleto.

Las biopsias de cada caso se obtuvieron del archivo de anatomía patológica, seleccionando las de mayor contenido tumoral y mayor indiferenciación. Se escogieron los bloques de parafina correspondientes a las laminillas previamente seleccionadas y a cada bloque se le practicaron 5 cortes: uno inicial de $5 \mu \mathrm{m}, 3$ de $50 \mu \mathrm{m}$ y finalmente otro de $5 \mu \mathrm{m}$. Los cortes de $5 \mu \mathrm{m}$ se tiñeron con hematoxilina-eosina y se confirmó la existencia de células tumorales en ambos. En el primer corte se estudió el grado histológico utilizando la puntuación de Gleason, lo que realizó un único patólogo. Para el análisis estadístico agrupamos a los tumores en dos grupos: Gleason 2 a 7 y Gleason 8 a 10.

Las secciones de $50 \mu \mathrm{m}$ se utilizaron para analizar el contenido de ADN mediante citometría de flujo. La preparación de las suspensiones nucleares a partir de las muestras parafinadas se realizó utilizando la técnica descrita por Hedley ${ }^{5}$. Una vez desparafinado el tejido se obtuvieron las células mediante una técnica previamente descri$\mathrm{ta}^{6}$. Para la tinción específica con yoduro de propidio el sedimento nuclear obtenido fue procesado mediante el kit comercial: DNA-Prep (Coulter Reagents Kit) que está compuesto de 2 preparaciones: DNA-Prep LPR, y DNA Prep. Stain. 
El material procesado fue adquirido y analizado mediante un citómetro de flujo Cytoronabsolute (Ortho Diagnostic Systems Inc.). Sólo se han aceptado aquellos histogramas con un coeficiente de variación inferior a 10. Los tumores fueron considerados como diploides, aneuploides hiperdiploides y tetraploide, cuando el índice de ADN fue igual a $1,1,05-1,89$ y 1,9-2,1, respectivamente $^{6}$. Para el análisis estadístico los tumores tetraploides e hiperdiploides se unieron en un solo grupo.

Para el estudio de morfometría nuclear se utilizaron los cortes de $5 \mu \mathrm{m}$ teñidos con hematoxilina-eosina. La medición se realizó utilizando un objetivo de 100 aumentos con aceite de inmersión en un microscopio óptico marca Nikon, proporcionando un aumento de 1400. El microscopio se conectó a una cámara de video que a su vez está conectada a un ordenador personal. Las mediciones se realizaron de modo interactivo con un planímetro constituido por un tablero digitalizado (marca Synoptics) conectado a un ordenador PC, dotado con el programa informático modelo INSIGHT-PC. Se midieron 90 núcleos por biopsia. Se desecharon todas aquellas células que no poseyeran una cromatina y membrana nuclear intactas, así como núcleos hipercromáticos, picnóticos, núcleos de células superpuestas y los núcleos en cortes oblicuos. El programa informático calcula automáticamente los diversos parámetros geométricos, obteniendo hasta 16 descriptores morfométricos con 17 medidas estadísticas para cada uno de ellos, con lo que se puede disponer hasta de 272 parámetros por núcleo.

Estudiamos 14 descriptores morfométricos: área nuclear $\left(\mu m^{2}\right)$, eje $X$ de elipticidad $(\mu m)$, eje $Y$ de elipticidad $(\mu \mathrm{m})$, anchura máxima $(\mu \mathrm{m})$, longitud máxima $(\mu \mathrm{m})$, perímetro: corresponde a la circunferencia nuclear $(\mu \mathrm{m})$, factor de forma (factor de redondez y regularidad de la superficie), orientación celular (expresa la polaridad celular), centro X de gravedad (ejes celulares en función de la tendencia central gravitatoria), centro $\mathrm{Y}$ de gravedad (ejes celulares en función de la tendencia central gravitatoria), índice axial (anchura máxima/longitud máxima), cociente área/estructura global seleccionada (\%), diámetro de la esfera $(\mu \mathrm{m})$, volumen de la esfera $\left(\mu \mathrm{m}^{3}\right)$.
Un coeficiente de variación menor del 5\% intra e interinvestigador demostró la exactitud y reproductibilidad de las medidas.

Para eliminar la información redundante que aporta el estudio de morfometría nuclear, se realizó un estudio de correlación, seleccionando únicamente aquellas variables que aportaran información independiente. Las variables seleccionadas fueron estratificadas para el estudio estadístico.

Los factores pronósticos incluidos en el análisis fueron: la edad, la categoría $\mathrm{T}$, el hematocrito, el nivel de albúmina sanguínea, las fosfatasas alcalinas, las fosfatasas ácidas prostáticas, la puntuación de Gleason, el resultado del rastreo óseo, la ploidía del ADN y los parámetros morfométricos que aportaron información independiente.

Se realizaron estudios de supervivencia univariante para el total de la muestra y para cada uno de los factores pronósticos mediante el método de Kaplan-Meier, calculando el riesgo relativo de cada uno de los grupos. Como técnica de análisis multivariante se utilizó el método de regresión múltiple de riesgos proporcionales de Cox. Como variables explicativas o predictoras se han considerado las características clínicas, anatomopatológicas y los parámetros derivados del estudio de contenido en ADN y morfometría nuclear. Como variable respuesta se ha considerado el estado de vivo o muerto.

Para saber si las variables experimentales (ADN y morfometría nuclear) aportan suficiente información pronóstica adicional a los factores clásicos (edad, estadio $\mathrm{T}$, hematocrito, nivel de albúmina, fosfatasas alcalinas, fosfatasas ácidas prostáticas, Gleason y rastreo óseo) que justifique su utilización, realizamos un análisis de regresión logística cuya variable respuesta es que el paciente alcance o no la mediana de supervivencia de la muestra. Calculamos la sensibilidad y la especificidad del análisis únicamente con los factores pronósticos clásicos y añadiendo los factores pronósticos experimentales.

\section{RESULTADOS}

La edad media de los pacientes fue de $69( \pm 8)$ años. En la Tabla I se describen las características clínicas e histológicas de los pacientes, así como el resultado del análisis de supervivencia univariante, con sus riesgos relativos. La mediana 
TABLA I

DISTRIBUCIÓN DE LA MUESTRA POR EDAD, ESTADIO LOCAL, HEMATOCRITO, NIVEL DE ALBÚMINA, FOSFATASAS ALCALINAS Y FOSFATASAS ÁCIDAS SÉRICAS, PUNTUACIÓN DE GLEASON Y RESULTADO DEL RASTREO ÓSEO. RESULTADO DEL ANÁLISIS UNIVARIANTE DE SUPERVIVENCIA

\begin{tabular}{|c|c|c|c|c|c|}
\hline Variable & $\begin{array}{l}\text { Número de } \\
\text { casos (\%) }\end{array}$ & $\begin{array}{l}\text { Mediana de } \\
\text { supervivencia } \\
\text { en meses }\end{array}$ & $\begin{array}{c}\text { R.R.* }^{*} \\
\text { (test de Cox) }\end{array}$ & I.C. $95 \%^{+}$ & Valor de $\mathbf{P}$ \\
\hline \multicolumn{6}{|l|}{ EDAD } \\
\hline$\leq 65$ & $17(31,5)$ & 25 & 1 & & \multirow{3}{*}{0,9} \\
\hline $66-75$ & $26(48,1)$ & 31 & 1,04 & $0,50-2,14$ & \\
\hline$>75$ & $36(20,4)$ & 24 & 1,01 & $0,41-2,47$ & \\
\hline \multicolumn{6}{|l|}{ CATEGORİA T } \\
\hline $\mathrm{T} 2$ & $2(3,7)$ & - & - & & \multirow{3}{*}{0,027} \\
\hline T3 & $27(50,0)$ & 43 & 1 & & \\
\hline $\mathrm{T} 4$ & $25(46,3)$ & 21 & 2,02 & $1,07-3,83$ & \\
\hline \multicolumn{6}{|l|}{ HEMATOCRITO } \\
\hline Normal & $35(64,8 \%)$ & 31 & 1 & & \multirow{2}{*}{0,33} \\
\hline Disminuido & $18(35,2 \%)$ & 26 & 1,39 & $0,71-2,71$ & \\
\hline \multicolumn{6}{|l|}{ ALBÜMINA } \\
\hline Normal & 41 (75,9\%) & 33 & 1 & & \multirow{2}{*}{$<0,001$} \\
\hline Disminuida & $13(24,1 \%)$ & 11 & 3,6 & $1,78-7,31$ & \\
\hline \multicolumn{6}{|c|}{ FOSFATASAS ALCALINAS } \\
\hline Normales & 31 (57\%) & 43 & 1 & & \multirow{2}{*}{0,019} \\
\hline Aumentadas & $23(43 \%)$ & 21 & 2,06 & $1,10-3,82$ & \\
\hline \multicolumn{6}{|c|}{ FOSFATASAS ÁCIDAS PROSTÁTICAS } \\
\hline Normales & 27 (50\%) & 24 & 1 & & \multirow{2}{*}{0,634} \\
\hline Aumentadas & 27 (50\%) & 30 & 0,86 & $0,46-1,59$ & \\
\hline \multicolumn{6}{|c|}{ PUNTUACIÓN DE GLEASON } \\
\hline Gleason 2-4 & $5(9 \%)$ & 55 & 1 & & \multirow{3}{*}{$<0,001$} \\
\hline Gleason 5-7 & 29 (54\%) & 38 & 1,16 & $0,39-3,45$ & \\
\hline Gleason 8-10 & 20 (37\%) & 13 & 3,67 & $1,21-11,09$ & \\
\hline \multicolumn{6}{|l|}{ RASTREO ÓSEO } \\
\hline 1-6 Focos & $34(2,9 \%)$ & 30 & 1 & & \multirow{3}{*}{0,035} \\
\hline$>6$ focos & $13(24,1 \%)$ & 38 & 0,91 & $0,43-1,93$ & \\
\hline > 75\% del esqueleto & $7(13 \%)$ & 19 & 2,81 & $1,18-6,72$ & \\
\hline
\end{tabular}

*R.R.: Riesgo Relativo. +'I.C. 95\%: Intervalo confianza al 95\%.

de supervivencia global fue de 30 meses y la probabilidad de sobrevivir a los 5 y 10 años del $25 \%$ y $10 \%$, respectivamente, (Fig. 1). Casi todos los pacientes tenían tumores localmente avanzados (T3 y T4). En cuanto a las características analíticas, el 35\% tenían el hematocrito inferior al normal, el
$24 \%$ mostraban disminución de la albúmina sanguínea, el $43 \%$ presentaban elevadas las fosfatasas alcalinas y la mitad las fosfatasas ácidas prostáticas. En el análisis univariante de supervivencia (Tabla I), entre las variables clínicas e histológicas mostraron tener carácter pronóstico 


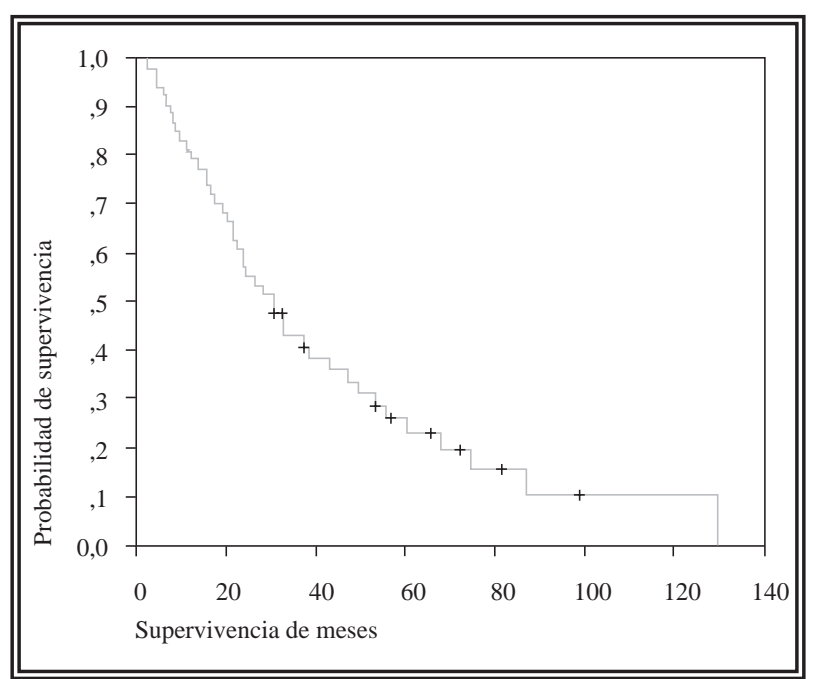

FIGURA 1. Curva de supervivencia global para toda la muestra.

la categoría T, la concentración de albúmina sanguínea, las fosfatasas alcalinas séricas, la puntuación de Gleason (cuando estaba entre 8 y 10), y el rastreo óseo (más del 75\% del esqueleto afecto). La edad, el hematocrito y las fosfatasas ácidas prostáticas no mostraron influencia significativa en la supervivencia.

En cuanto al estudio de ADN, 8 muestras no fueron válidas para el estudio. De las válidas, el $63 \%(29 / 46)$ fueron diploides, el 30\% (14/46) y el $7 \%(3 / 46)$ aneuploides tetraploides. Para el análisis de datos hemos incluido los tumores tetraploides en el grupo de los aneuploides hiperdiploides. En la Figura 2 se observa el resultado del análisis de supervivencia univariante, con los correspondientes riesgos relativos expresados en la Tabla II. Observamos que la alteración en la ploidía del ADN es un factor de mal pronóstico para la supervivencia.

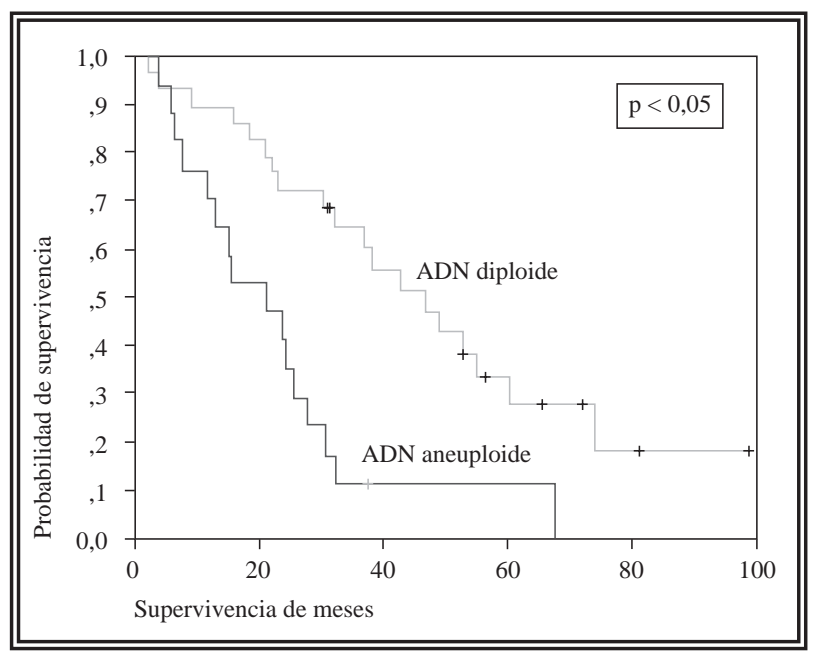

FIGURA 2. Análisis de supervivencia según el resultado del estudio de ADN mediante citometria de flujo.

Con el estudio de morfometría estudiamos 14 descriptores nucleares. Un estudio de correlación nos permitió eliminar aquellos con información redundante, siendo 5 los parámetros que aportan toda la información. De ellos, únicamente el área nuclear media presenta valor pronóstico en el estudio univariante de supervivencia (Tabla III), de tal forma que aquellos pacientes cuyas células tumorales tienen un área nuclear media mayor de $45 \mu \mathrm{m}^{2}$ presentan menor supervivencia que el resto (Fig. 3).

En el estudio multivariante de supervivencia se introdujeron como variables explicativas la edad, la categoría $\mathrm{T}$, el hematocrito, el nivel de albúmina sanguínea, el nivel de fosfatasas alcalinas y fosfatasas ácidas prostáticas, la puntuación de Gleason, el resultado del rastreo, la ploidía del ADN y el área nuclear media y como variable respuesta el estado de vivo o muerto al cerrar

\section{TABLA II}

RESULTADO DEL ESTUDIO DE CITOMETRÍA DE FLUJO, CON EL ANÁLISIS DE SUPERVIVENCIA UNIVARIANTE. SE HAN AGRUPADO LOS TUMORES ANEUPLOIDES HIPERDIPLOIDES Y TETRAPLOIDES

\begin{tabular}{|l|c|c|c|c|c|}
\hline Ploidia del ADN & $\begin{array}{c}\text { Número de } \\
\text { casos (\%) }\end{array}$ & $\begin{array}{c}\text { Mediana de } \\
\text { supervivencia } \\
\text { en meses }\end{array}$ & $\begin{array}{c}\text { R.R.* } \\
\text { (test de } \\
\text { Cox) }\end{array}$ & I.C. 95\% $^{+}$ & $\begin{array}{c}\text { Valor de P } \\
\text { (Long-Rank) }\end{array}$ \\
\hline ADN diploide & $29(63 \%)$ & 47 & 1 & $1,52-6,22$ & 0,001 \\
\hline ADN aneuploide & $17(37 \%)$ & 21 & 3,08 & \multirow{2}{*}{} \\
\hline
\end{tabular}

*R.R. Riesgo Relativo.

+ I.C. 95\%.: Intervalo confianza al 95\%. 
TABLA III

DISTRIBUCIÓN DE LA MUESTRA SEGÚN LAS VARIABLES MORFOMÉTRICAS ESTRATIFICADAS QUE OFRECEN INFORMACIÓN INDEPENDIENTE SEGÚN EL ESTUDIO DE CORRELACIÓN: ÁREA NUCLEAR MEDIA, FACTOR DE FORMA, ORIENTACIÓN, CENTRO DE GRAVEDAD X Y CENTRO DE GRAVEDAD Y

\begin{tabular}{|c|c|c|c|c|c|}
\hline Variable & $\begin{array}{l}\text { Número de } \\
\text { casos (\%) }\end{array}$ & $\begin{array}{c}\text { Mediana } \\
\text { supervivencia } \\
\text { en meses }\end{array}$ & $\begin{array}{l}\text { R.R.* } \\
\text { (test de } \\
\text { Cox) }\end{array}$ & I.C. $95 \%^{+}$ & $\begin{array}{l}\text { Valor de P } \\
\text { (Long-Rank) }\end{array}$ \\
\hline \multicolumn{6}{|c|}{ Área nuclear $\left(\mu \mathrm{m}^{2}\right)$} \\
\hline$<45$ & $30(55 \%)$ & 60 & 1 & & \multirow{2}{*}{$\mathrm{p}<0,001$} \\
\hline$\geq 45$ & $24(45 \%)$ & 15 & 6,07 & $2,88-12,75$ & \\
\hline \multicolumn{6}{|c|}{ Factor de forma (\%) } \\
\hline$\leq 87 \%$ & 24 (44\%) & 32 & 1 & & \multirow{2}{*}{$\mathrm{p}=0,24$} \\
\hline$>87 \%$ & $30(56 \%)$ & 23 & 1,46 & $0,77-2,76$ & \\
\hline \multicolumn{6}{|c|}{ Orientación } \\
\hline$<85$ & $31(57,4 \%)$ & 25 & 1 & & \multirow{2}{*}{$\mathrm{p}=0,72$} \\
\hline$\geq 85$ & $23(42,6 \%)$ & 30 & 1,11 & $0,60-2,07$ & \\
\hline \multicolumn{6}{|l|}{ Centro X } \\
\hline$<353$ & 23 (42,6\%) & 19 & 1 & & \multirow{2}{*}{$\mathrm{p}=0,49$} \\
\hline$\geq 353$ & $31(47,4 \%)$ & 32 & 0,80 & $0,43-1,49$ & \\
\hline \multicolumn{6}{|l|}{ Centro Y } \\
\hline$<280$ & $23(42,6 \%)$ & 23 & 1 & & \multirow{2}{*}{$\mathrm{p}=0,34$} \\
\hline$\geq 280$ & $31(47,4 \%)$ & 30 & 0,74 & $0,40-1,37$ & \\
\hline
\end{tabular}

*R.R.: Riesgo Relativo.

${ }^{+}$I.C. 95\%.: Intervalo de confianza al 95\%.

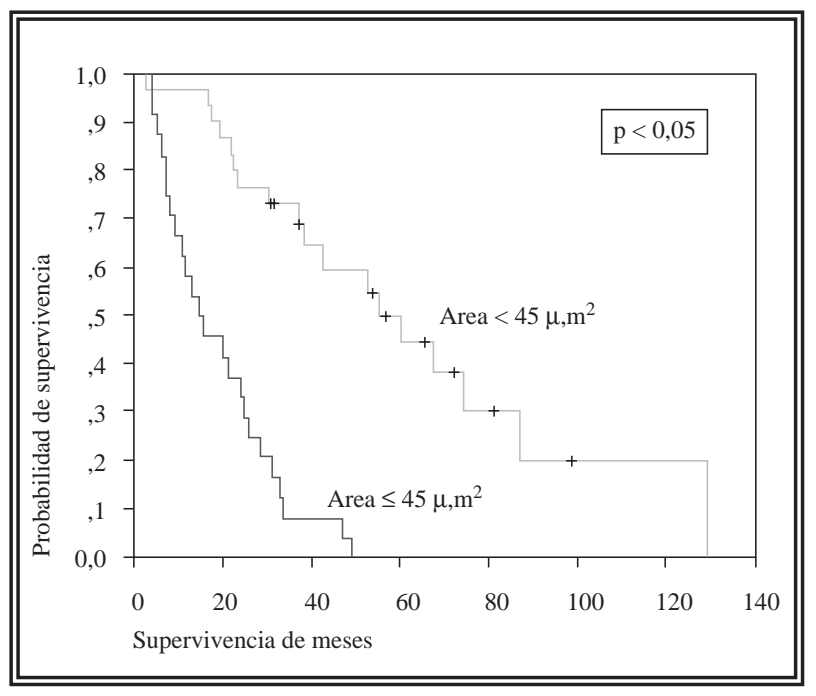

FIGURA 3. Análisis de supervivencia para el área nuclear media. el estudio. La regresión de Cox seleccionó como significativas la afectación de más del $75 \%$ del esqueleto en el rastreo óseo, la aneuploidía del $\mathrm{ADN}$, el área nuclear media mayor de $45 \mu^{2}$ y la puntuación de Gleason de 8 o superior, de tal forma que los pacientes con tumores aneuploides presentan un riesgo de muerte más de 3 veces superior a los diploides, al igual que los que tie-

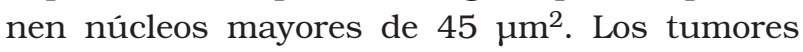
más indiferenciados (Gleason 8 o superior) presentan un riesgo de mortalidad de más del doble, mientras que aquellos cuyo rastreo óseo muestra afectación de más del 75\% del esqueleto el riesgo se multiplica por 9 (Tabla IV).

El análisis de regresión logística para los factores clásicos (Tabla V) selecciona únicamente la puntuación de Gleason como variables predictoras, 
TABLA IV

ANÁLISIS MULTIVARIANTE. MODELO DE REGRESIÓN OBTENIDO UTILIZANDO LAS VARIABLES: EDAD, CATEGORÍA T, HEMATOCRITO, NIVEL DE ALBÚMINA, FOSFATASAS ALCALINAS, FOSFATASAS ÁCIDAS PROSTÁTICAS, PUNTUACIÓN DE GLEASON, RESULTADO DEL RASTREO, ÁREA NUCLEAR MEDIA Y PLOIDÍA DEL ADN

\begin{tabular}{|c|c|c|c|c|}
\hline $\begin{array}{l}\text { Variable } \\
\text { seleccionada }\end{array}$ & Coeficiente B & $\begin{array}{c}\text { Riesgo } \\
\text { Relativo* }\end{array}$ & I.C. $95 \%^{+}$ & Valor de $p$ \\
\hline $\begin{array}{l}\text { Rastreo óseo } \\
\quad>6 \text { focos (1) } \\
75 \% \text { del esqueleto }\end{array}$ & $\begin{array}{l}0,2736 \\
2,2145\end{array}$ & $\begin{array}{l}1,31 \\
9,15\end{array}$ & $\begin{array}{c}0,55-3,14 \\
2,79-29,99\end{array}$ & $\begin{array}{c}0,001 \\
0,538 \\
<0,001\end{array}$ \\
\hline ADN aneuploide & 1,2749 & 3,57 & $1,38-9,28$ & 0,009 \\
\hline Área nuclear >45 $\mu \mathrm{m}$ & 0,9679 & 3,43 & $1,07-6,45$ & 0,034 \\
\hline Gleason 8-10 & 0,8774 & 2,40 & $1,07-5,39$ & 0,033 \\
\hline
\end{tabular}

*Test de Cox.

+Intervalo de confianza al 95\%.

\section{TABLA V}

RESULTADO DEL ANÁLISIS DE REGRESIÓN LOGÍSTICA PARA LOS FACTORES PRONÓSTICOS CLÁSICOS (EDAD, ESTADIO T, RASTREO ÓSEO, NIVEL DE ALBÚMINA, FOSFATASAS ÁCIDAS, FOSFATASAS ALCALINAS, HEMATOCRITO Y PUNTUACIÓN DE GLEASON) Y CUANDO SE AÑADEN LOS EXPERIMENTALES (PLOIDÍA DEL ADN Y ÁREA NUCLEAR MEDIA). LA VARIABLE RESPUESTA EN AMBOS CASOS ES QUE EL PACIENTE ALCANCE LA MEDIANA DE SUPERVIVENCIA (30 MESES)

\begin{tabular}{|c|c|c|c|c|}
\hline Variables seleccionadas & Coeficiente B & O.R.* & (I.C. 95\%) ${ }^{+}$ & Valor de $\mathbf{p}$ \\
\hline \multicolumn{5}{|c|}{ Factores pronósticos clásicos } \\
\hline Gleason entre 8 y 10 & 2,6 & 14,1 & $3,1-63,7$ & $<0,001$ \\
\hline \multicolumn{5}{|c|}{ Factores pronósticos clásicos y experimentales } \\
\hline Gleason entre 8 y 10 & 2,9 & 18,9 & $2,5-144,6$ & 0,005 \\
\hline Rastreo óseo (>75\%) & 2,7 & 16,1 & $1,4-186,1$ & 0,026 \\
\hline $\mathrm{ADN}$ aneuploide & 2,7 & 15,5 & $2,0-119,5$ & 0,008 \\
\hline
\end{tabular}

*O.R.: Odds Ratio

+I.C. 95\%.: Intervalo de confianza al 95\%.

para saber si un paciente alcanzará la mediana de supervivencia (30 meses) con una sensibilidad del $89,3 \%$ y una especificidad del $64 \%$. Cuando se incluyen las variables experimentales, ADN y morfometría nuclear, el análisis selecciona la puntuación de Gleason, el rastreo óseo (afectación de más del $75 \%$ del esqueleto) la ploidía del ADN, con una sensibilidad del $90 \%$ y especificidad del $72 \%$.

\section{DISCUSIÓN}

La supervivencia de los pacientes con cáncer de próstata metastásico es extremadamente variable. En general, la mediana de supervivencia después del diagnóstico de la enfermedad metastásica oscila entre 2 y 3 años $^{7}$, aunque puede variar desde meses hasta más de 10 años $^{8,9}$. Diversos parámetros clínicos y de laboratorio han sido identificados como predictores del comportamiento tumoral cuando ya se han producido las metástasis. Se ha descrito el valor pronóstico del grado de afectación del esqueleto, estudiado tanto radiológicamente ${ }^{10}$ como mediante rastreo óseo ${ }^{11-}$ 14. Nosotros únicamente encontramos que tenga influencia en el pronóstico cuando estamos ante una afectación masiva del esqueleto ("superscan"), lo que determinará una mala respuesta al tratamiento en términos de supervivencia. 
Existen una serie de marcadores bioquímicos, cuya importancia pronóstica se ha descrito, como es el caso de los niveles séricos de fosfatasas alcalinas $^{14-20}$, que si bien en nuestra serie mostraron valor pronóstico en el estudio univariante, son excluidos en el multivariante. Por el contrario, coincidimos con otros autores en la escasa utilidad de la fosfatasa ácida prostática ${ }^{12,16-19}$. También la anemia se ha revelado como factor pronóstico en la mayoría de estudios ${ }^{14,15,17-19,21}$. Sin embargo, en nuestra serie carece de valor, tanto en el análisis univariante como en el multivariante. El estado nutricional del paciente, determinado por el nivel de albúmina, ha sido poco estudiado. En nuestra serie sí que mostró carácter pronóstico en el estudio univariante, pero no en el multivariante.

Observamos que el estadio local no influye en la supervivencia en el cáncer de próstata metastásico 22 , a pesar de que hay estudios que le atribuyen valor pronóstico ${ }^{15}$. Se ha reportado que el grado histológico, un potente factor pronóstico, pierde casi todo su valor cuando estamos ante un paciente con metástasis ${ }^{22}$. Sin embargo algunos estudios, al igual que el nuestro, describen cierta capacidad del grado para predecir el comportamiento tumoral ${ }^{15,23,24}$. En nuestro estudio, tanto en el análisis univariante como el multivariante, la indiferenciación tumoral (puntuación de Gleason 8 a 10) incrementaba significativamente el riesgo de mortalidad.

Si bien se ha establecido la influencia de la ploidía en los tumores tratados hormonalmen$\mathrm{te}^{25,26}$, no se ha definido definitivamente su valor pronóstico y aunque hay estudios que indican que la ploidía del tumor primario carece de importancia pronóstica cuando existen metástasis esqueléticas ${ }^{22,27,28}$, otros, al igual que el nuestro, señalan cierta influencia. Así Miller y cols. ${ }^{23}$ en una serie de pacientes con tumores estadio D2 sometidos a bloqueo androgénico, describen que el contenido en ADN presenta valor predictivo en términos de supervivencia y ofrece una potente información pronóstica cuando se asocia al grado. También se ha descrito su influencia en la respuesta al bloqueo androgénico adyuvante tras prostatectomía radical en pacientes en estadio $\mathrm{D} 1^{29}$. Sin embargo, observamos que mejora escasamente la capacidad de los factores pronósticos clásicos para predecir si el paciente alcanzará la mediana de supervivencia.

La mayoría de estudios de morfometría se han realizado en pacientes con tumores clínicamente localizados y aunque en algunos casos se observa relación entre determinados parámetros morfométricos y el pronóstico ${ }^{24,30-33}$, son escasos los estudios que intenten establecer la utilidad de la morfometría en el cáncer de próstata metastásico, Fujikawa y cols. refieren el valor del volumen nuclear medio como valor pronóstico independiente cuando analiza los pacientes con metástasis ${ }^{24}$. También cuando Zhang y cols. estudian por separado los pacientes con tumores en estadio $\mathrm{D}$, el volumen nuclear medio es un parámetro morfométrico con influencia pronóstica tanto en el estudio univariante como en el multivariante ${ }^{34}$. En nuestra serie, la morfometría es seleccionada como factor pronóstico independiente, concretamente el valor del área nuclear media, de tal forma que el riesgo de mortalidad, al igual que con la aneuploidía, se triplica cuando el área nuclear media es superior a los $45 \mu^{2}$ (Tabla IV). En nuestro caso, es el área nuclear media el factor significativo. No estudiamos la influencia de otros parámetros morfométricos, por la alta correlación existente entre ellos. Así el volumen nuclear medio, cuyo valor ha sido reportado recientemen$t^{24,33}$, está altamente correlacionado con el área $(\mathrm{r}=0,93 ; \mathrm{p}<0,01)$ por lo que el significado pronóstico debe ser el mismo. Es por ello que consideramos, al igual que otros ${ }^{34}$, que su utilización no aporta información adicional al área nuclear media. Por otra parte, el área nuclear no es seleccionada en el estudio de regresión logística, con lo que en la balanza coste-eficacia se hace cuestionable su utilidad predictiva en estos pacientes.

Así pues, aunque existen estudios que no son concluyentes, sí que podemos afirmar que el estudio de las características del tumor primario puede aportar información sobre la posible evolución del enfermo con cáncer de próstata metastásico. Sin embargo, aunque la ploidía del ADN y la morfometría nuclear aporta información pronóstica independiente en el estudio de supervivencia, el coste y la complejidad de su realización no justifica su utilización al mejorar escasamente la capacidad predictiva de los factores pronósticos clásicos. 


\section{REFERENCIAS}

1. BRAWN PN.: The dedifferentiation of prostate carcinoma. Cancer 1983; 52: 246-251.

2. VAN DEN OUDEN D, TRIBUKAIT B, BLOM JH et al.: Deoxyribonucleic acid ploidy of core biopsies and metastatic lymph nodes of prostate cancer patients: impact on time to progression. The European Organization for Research and Treatment of Cancer Genitourinary Group. J Urol 1993; 150: 400-406.

3. BABIARZ J, PETERS JM, MILES B, CRISSMAN JD.: Comparison of DNA content in primary and lymph node metastases in prostate adenocarcinoma. Anal Quant Cytol Histol 1993; 15: 158-164.

4. SOBIN LH, WITTEKIND CH (eds).: UICC: TNM classification of malignant tumours, 5th edn. New York. Wiley-Liss, Inc. 1997.

5. HEDLEY WD, FRIEDLANDER ML, TAYLOR IW, RUGG CA, MUSGROVE EA.: Method for analysis of cellular DNA content of paraffin-embedded pathological material using flow cytometry. $J$ Histochem Cytochem 1983; 31: 1333-1335.

6. RUIZ CERDÁ JL, HERNÁNDEZ M, GOMIS F et al.: Value of deoxyribonucleic acid ploidy and nuclear morphometry for prediction of disease progression in renal cell carcinoma. J Urol 1996; 155: 459-465.

7. ROBSON M, DAWSON N.: How is androgen-dependent mestastatic prostate cancer best treated? Hematol Oncol Clin North Am 1996; 10: 727-747.

8. JORDAN WP Jr, BLACKARD CE, BYAR DP.: Reconsideration of orchiectomy in the treatment of advanced prostatic carcinoma. South Med J 1977; 70: 1411-1413.

9. DEARNALEY DP.: Cancer of the prostate. BMJ 1994; 308: 780-784.

10. HOVSEPIAN JA, BYAR DP, and the Veterans Administration Cooperative Urological Research Group.: Carcinoma of the prostate: correlation between radiologic quantitation of metastases and patient survival. Urology 1975; 6: 11-16.

11. SOLOWAY MS, HARDEMAN SW, HICKEY D et al.: Stratification of metastatic prostate cancer based on extent of disease on initial bone scan. Cancer 1988; 61: 195-202.

12. ISHIKAWA S, SOLOWAY MS, VAN DER ZWAAG R, TODD B.: Prognostic factors in survival free of progression after androgen deprivation therapy for treatment of prostate cancer. J Urol 1989; 141: 1139-1142.

13. SOLOWAY MS.: The importance of prognostic factors in advanced prostate cancer. Cancer 1990; 66: 1017-1021. Ernst DS, Hanson J, Venner PM. Analysis of prognostic factors in men with metastatic prostate cancer. $J$ Urol 1991; 146: 372-376.

14. EISENBERGER MA, CRAWFORD ED, WOLF M et al.: Prognostic factors in stage D2 prostate cancer: important implications for future trials: results of a Cooperative Intergroup Study (INT.0036). Semin Oncol 1994; 21: 613-619.

15. DE VOOGT HJ, SUCIU S, SILVESTRE R, PAVONEMACALUSO M, SMITH PH, DE PAUW $M$, and Members of the European Organization for Research on Treatment of Cancer Genitourinary
Tract Cancer Cooperative Group: multivariate analysis of prognostic factors in patients with advanced prostatic cancer: results from 2 european organization for research on treatment of cancer trials. J Urol 1989; 141: 883-888.

16. CHODAK GW, VOGELZANG NJ, CAPLAN RJ, SOLOWAY M, SMITH JA.: Independent prognostic factors in patients with metastatic (stage D2) prostate cancer. The Zoladex study group. JAMA 1991; 265: 618-621.

17. MULDERS PF, DIJKMAN GA, FERNÁNDEZ DEL MORAL P, THEEUWES AG, DEBRUYNE FM.: Analysis of prognostic factors in disseminated prostatic cancer. An update. Cancer 1990; 65: 27582761.

18. MATZKIN H, SOLOWAY MS, SCHELLHAMMER PF et al.: Prognostic factors in stage D2 prostate cancer treated with a pure nonsteroidal antiandrogen. Cancer 1993; 72: 1286-1290.

19. JORGENSEN T, KANAGASINGAM Y, KAALHUS O et al.: Prognostic factors in patients with metastatic (stage D2) prostate cancer: experience from the Scandinavian prostatic cancer group study-2. $J$ Urol 1997; 158: 164-170.

20. SMITH JA, LANGE PH, JANKNEGT RA, ABBOU CC, DEGERY A.: Serum markers as a predictor of response duration and patient survival after hormonal therapy for metastatic carcinoma of the prostate. J Urol 1997; 157: 1329-1334.

21. EMRICH LJ, PRIORE RL, MURPHY GP, BRADY MF.: Prognostic factors in patients with advanced stage prostate cancer. Cancer Res 1985; 45: 5173 5179.

22. JORGENSEN T, YOGESAN K, SKJORTEN F, BERNER A, TVETER KJ, DANIELSEN HE. Histopathological grading and DNA ploidy as prognostic markers in metastatic prostatic cancer. $\mathrm{Br} J$ Cancer 1995; 71: 1055-1060.

23. MILLER J, HORSFALL DJ, MARSHALL VR, RAO DM, LEONG SY.: The prognostic value of deoxyribonucleic acid flow cytometric analysis in stage D2 prostatic carcinoma. J Urol 1991; 145: 11921196.

24. FUJIKAWA K, SASAKI M, ARAI Y, YAMABE $H$, OGAWA O, YOSHIDA O.: Prognostic criteria in patients with prostate cancer: Gleason score versus volume-weighted mean nuclear volume. Clin Cancer Res 1997; 3: 613-618.

25. FORSSLUND G, ESPOSTI P, NILSSON B, ZETTERBERG A.: The prognostic significance of nuclear DNA content in prostatic carcinoma. Cancer 1992; 69: 1432-1439.

26. TRIBUKAIT B.: Nuclear deoxyribonucleic acid determination in patients with prostate carcinomas: clinical research and application. Eur Urol 1993; 23 (suppl 2): 64-76.

27. YOKOGI H, MIZUTANI M, ILSHIBE T.: Flow Cytometric DNA analysis of stage D2 prostatic carcinoma. Urol Int 1991; 47: 57-59.

28. HEDLUND PO, ESPOSTI P, FALKMER U, JACOBSSON H, and participants in the SPCG-I study: DNA as a prognostic marker in advanced highgrade prostatic cancer. Acta Oncol 1991; 30: 215217. 
29. ZINCKE H, BERGSTRALH EJ, LARSON-KELLER JJ et al.: Stage D1 prostate cancer treated by radical prostatectomy and adjuvant hormonal treatment. Evidence for favourable survival in patients with DNA diploid tumors. Cancer 1992; 70 (1 Suppl): 311-323.

30. PARTIN AW, STEINBERG GD, PITCOCK RV et al.: Use of nuclear morphometry, Gleason histologic scoring, clinical stage and age to predict diseasefree survival among patients with prostate cancer. Cancer 1992; 70: 161-168.

31. VELTRI RW, PARTIN AW, EPSTEIN JE et al. Quantitative nuclear morphometry, Markovian texture descriptors, and DNA content captured on a CAS-200 image analysis system, combined with PCNA and HER-2/neu immunohistochemistry for prediction of prostate cancer progression. J Cell Biochem Suppl 1994; 19: 249-258.

32. VESALAINEN S, NORDLING S, LIPPONEN P, TALJA M, SYRJANEN K.: Progression and survival in prostatic adenocarcinoma a comparison of clinical stage, Gleason grade, S-phase fraction an DNA ploidy. Br J Cancer 1994; 70: 309-314.
33. ARIMA K, SUGIMURA Y, HIOKI T, YAMASHITA A, KAWAMURA.: Stereologically estimated mean nuclear volume of prostatic cancer is a reliable prognostic parameter. Br J Cancer 1997; 76: 234237.

34. ZHANG YH, KANAMARU $\mathrm{H}$, OYAMA $\mathrm{N}$ et al.: Prognostic value of nuclear morphometry on needle biopsy from patients with prostate cancer: is volume-weighted mean nuclear volume superior to other morphometric parameters? Urology 2000; 55 377-381.

Dr. J.M. Martínez Jabaloyas

Servicio de Urología

Hospital Clínico Universitario

Avda. Blasco Ibáñez, 17

47010 Valencia

(Trabajo recibido el 17 octubre 2003) 